\title{
USO DE MESA VERTICAL COMO PARÂMETRO PARA REGULAGENS DE TURBOATOMIZADORES
}

\author{
RENILDO L. MION ${ }^{1}$, WEBERTE A. SOMBRA ${ }^{2}$, JOSÉ M. DE L. DUARTE ${ }^{3}$, ELIVÂNIA M. \\ S. NASCIMENTO ${ }^{3}$, CARLOS A. VILIOTTI ${ }^{1}$, FIDEL C. B. LUCAS ${ }^{2}$, CLESSON A. MONTE ${ }^{2}$
}

RESUMO: O consumo excessivo de agrotóxicos na agricultura brasileira é preocupante, e um dos fatores que contribuem para este excesso é o uso incorreto dos equipamentos de aplicação, causando grandes problemas de contaminação ambiental. O sucesso de uma aplicação agrícola somente é efetivado quando se consegue atingir o alvo com a menor contaminação ambiental possível. O objetivo deste trabalho foi comparar o perfil da distribuição vertical de um turboatomizador com e sem fluxo de ar e o número de bicos utilizados nos ramais, utilizando-se de uma mesa vertical como parâmetro de avaliação. O conjunto utilizado foi um trator marca VALTRA, modelo BM-120 4x2 TODA, e um turboatomizador da marca Jacto, modelo ARBUS 400 GOLDEN, com pontas do tipo J5-2, pressão de $1378 \mathrm{kPa}$ e velocidade do ar de $35 \mathrm{~m} \mathrm{~s}^{-1}$. O número de bicos não influenciou no perfil de distribuição volumétrico. O fluxo de ar influenciou no perfil de distribuição volumétrico tanto para o lado direito quanto para o lado esquerdo. Os maiores volumes ocorreram abaixo de $1,16 \mathrm{~cm}$ com o turboatomizador utilizando ou não o fluxo de ar, com 12 ou 6 pontas.

PALAVRAS-CHAVE: mesa vertical, acurácia, tecnologia de aplicação.

\section{THE USE OF A VERTICAL PATTERNATOR AS PARAMETER FOR ADJUSTMENTS OF AIR ASSITED SPRAYER}

\begin{abstract}
Excessive consumption of pesticides in Brazilian agriculture is of concern, and one of the factors contributing to this surplus is the incorrect use of equipment for its applying, causing severe environmental contamination. The success of agricultural applications is effective only when it manages to hit the target with the lowest possible environmental contamination. The aim of this study was to compare the profile of the vertical distribution of an air assisted sprayer with and without air flow and the number of nozzles used in extensions, using a vertical patternator as the parameter. The set used was a Valtra tractor, model BM-120 4x2 TDA and one Jacto air assisted sprayer jet, model ARBUS 400 GOLDEN, with nozzle J5-2, and pressure of $1378 \mathrm{kPa}$, with air velocity of $35 \mathrm{~m} \mathrm{~s}^{-1}$ at the time of the test was conducted. The number of nozzles did not influence the volumetric distribution profile. The airflow influenced the volumetric distribution profile for both right and left side. The largest volumes were below $1,16 \mathrm{~m}$ for the air assisted sprayer using or not the air flow, with 12 or 6 nozzles.
\end{abstract}

KEYWORDS: vertical patternator, accuracy, application technology.

\section{INTRODUÇÃO}

O consumo de agrotóxicos na agricultura brasileira é preocupante, destacando-se mundialmente e em franca expansão. O Brasil é responsável por 50\% da quantidade de agrotóxicos utilizados na América Latina, ocupando o terceiro lugar no ranking mundial de consumo destes insumos (SINDAG, 2009).

O sucesso de um programa de tratamento fitossanitário na agricultura depende fundamentalmente de produto com eficácia comprovada e de tecnologia desenvolvida para sua

\footnotetext{
${ }^{1}$ Eng $^{\circ}$ Agrícola, Prof. Adjunto, Departamento de Engenharia Agrícola, DENA/UFC, Fortaleza - CE, rmion@ufc.br.

${ }^{2}$ Graduando em Agronomia, UFC, Fortaleza - CE.

${ }^{3}$ Pós-graduando em Engenharia Agrícola (Eng. de Sistemas Agrícolas), DENA/UFC, Fortaleza - CE.

Recebido pelo Conselho Editorial em: 10-9-2010

Aprovado pelo Conselho Editorial em: 22-2-2011
} 
aplicação. Dessa forma, é preciso que se estabeleçam métodos de controle das aplicações de defensivos, principalmente no que diz respeito às máquinas aplicadoras (ALVARENGA \& CUNHA, 2010).

Segundo HOLOWNICKI et al. (2000), os turboatomizadores são os mais utilizados em culturas arbustivas, por serem fáceis de operar e efetivos no controle de pragas e doenças. Entretanto, os autores salientam que a pulverização com turboatomizadores se caracteriza como um processo ineficiente, devido às perdas ultrapassarem mais da metade dos produtos fitossanitários aplicados.

Os turboatomizadores tradicionais possuem ventilador montado atrás do pulverizador, com fluxo axial direcionado para cima e para fora. Os ventiladores de fluxo axial são projetados para movimentar grandes volumes de ar a baixas pressões, sendo que alguns pulverizadores possuem hélices de passo variável para fornecer fluxos de ar variáveis, dependendo da arquitetura, densidade de folhas e copa da cultura (LANDERS, 2002a). Para isso, LANDERS (2002b) desenvolveu uma placa de defletor ajustável para ser montada na base do topo e na saída de ar direcionando para o dossel da cultura, a qual melhorou a penetração e deposição de gotas em cerca de 20 a $30 \%$.

Devido ao sentido da rotação da turbina do turboatomizador, o movimento do ar sofre um desvio lateral à direita. Esse fato provoca maior direcionamento da calda de pulverização para o lado direito da parreira, considerando o sentido de deslocamento do trator (BALAN et al., 2006). FARROQ \& LANDERS (2004), avaliando a deposição de gotas em uma mesa vertical, constataram diferenças no volume entre os lados direito e esquerdo, devido ao fluxo de ar descendente e ascendente, respectivamente. De acordo com os autores, isso é devido ao sentido de rotação do ventilador do turboatomizador, o qual necessita de regulagem da angulação dos bicos nos ramais. PRATT et al. (2006) também realizaram observações semelhantes em que o lado direito do ventilador atingiu velocidade de $40,5 \mathrm{~m} \mathrm{~s}^{-1}$, e no lado esquerdo a velocidade foi de $32,8 \mathrm{~m} \mathrm{~s}^{-1}$.

LANDERS \& GIL (2006) constataram que a utilização de defletores em turboatomizadores foi mais eficaz em manter o fluxo de ar no sentido horizontal, coincidindo com o dossel da planta, em culturas arbóreas com alta densidade de folhas à penetração do ar no dossel da cultura diminuiu.

Os turboatomizadores são projetados para serem utilizados com a mesma configuração desde o início da cultura até ao final do seu ciclo, independentemente das mudanças no volume da copa e/ou densidade das folhas (LANDERS \& FAROOQ, 2004). As mudanças realizadas nos turboatomizadores estão relacionadas com o volume de aplicação da calda por área, entretanto não são feitas alterações relacionadas ao fluxo de ar (velocidade, volume ou direção). À medida que as culturas se desenvolvem, a densidade e o volume das folhas alteram-se sem a preocupação por parte dos agricultores quanto à deposição, frutos e o local em que as pragas e doenças se encontram. CUNNINGHAM \& HARDEN (1998) concluíram que os depósitos nas folhas da parte baixa da laranjeira foram superiores às da parte média e alta. Segundo o autor, a provável causa desta variação foi o fluxo gerado pelo ventilador, que produz uma distribuição desigual do fluxo na medida em que penetra na plantas e as folhas não conseguem ser totalmente cobertas.

PAI et al. (2009) constataram que a alta densidade da copa influencia na quantidade de ar que penetra no interior da planta, sendo necessário aumento do fluxo de ar para transportar as gotas até atingir o interior da copa das culturas. Segundo BALAN et al. (2006), o auxílio de ar propiciou melhor condição para maior depósito na aplicação de gotas maiores, menos sujeitas à deriva, o que promoveu o maior depósito para pontas com tamanho de gotas grandes, quando comparado ao uso de pontas com tamanho de gotas pequenas para o mesmo volume aplicado.

O objetivo do trabalho foi avaliar o perfil da distribuição vertical de um turboatomizador com e sem fluxo de ar e o número de bicos utilizados nos ramais, utilizando uma mesa vertical como parâmetro de avaliação. 


\section{MATERIAL E MÉTODOS}

O trabalho foi conduzido no Laboratório de Eletrônica e Máquinas Agrícolas (LEMA) e no Núcleo de Estudos de Máquinas Agrícolas do Semiárido (NEMASA) da Universidade Federal do Ceará, localizados em Fortaleza, Ceará, Brasil, nos meses de dezembro e janeiro de 2010.

Para a coleta do líquido pulverizado, foi construído um coletor de gotas constituído de um quadro de secção retangular com dimensões de 2,90 m de altura, 1,42 m de largura e com entalhes laterais a cada $21 \mathrm{~mm}$, onde foram montadas as calhas coletoras de plástico. As calhas coletoras são canais horizontais com 1,55 m de comprimento, de secção ondulada e ressalto interno longitudinal, conforme metodologia desenvolvida por DANTAS (2010). As laterais do quadro são abertas, e o coletor de gotas foi montado sobre uma estrutura de ferro com $1 \mathrm{~m}$ de comprimento e $0,18 \mathrm{~m}$ de largura, conforme Figura 1.

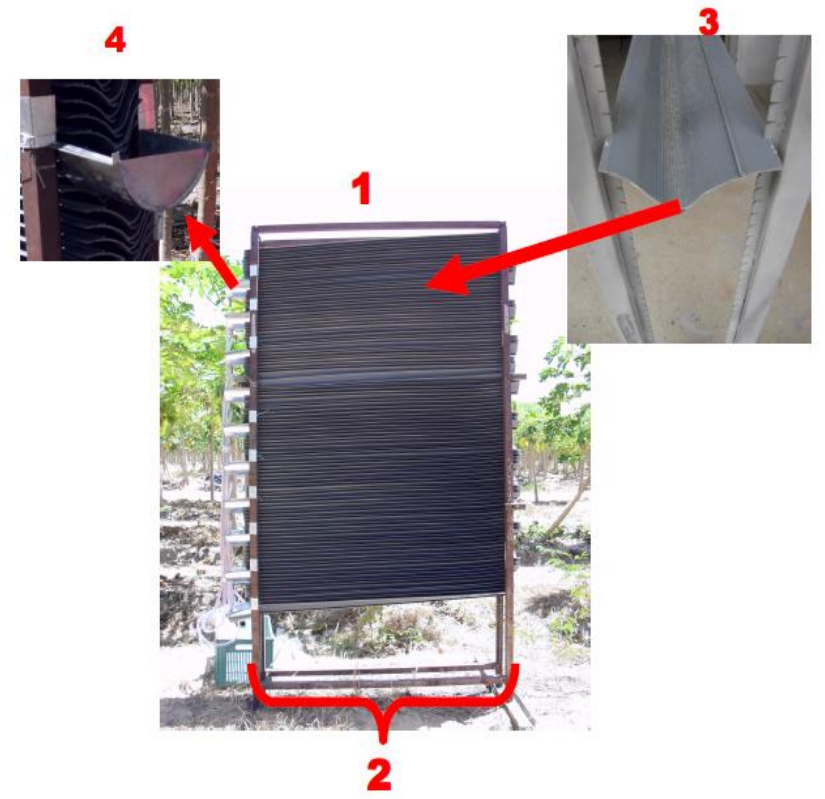

FIGURA 1. Vista frontal do coletor de gotas (1- mesa vertical; 2- estrutura de ferro; 3- lamela de plástico; 4- funil de zinco). Front view of the collector drops (1 - Vertical patternator, 2 - iron structure, 3 - plastic coverslip, 4 - zinc funnel).

$\mathrm{Na}$ estrutura principal, a cada dez calhas coletoras, foram instalados funis projetados para frente da área de captura do líquido pulverizado, funcionando como coletores da água captada nas calhas, responsáveis por conduzi-la até provetas graduadas, por meio de mangueiras de polietileno com diâmetro de $12,7 \mathrm{~mm}$, conectadas à saída do funil. As calhas coletoras foram posicionadas no interior da estrutura, com declividade de $12^{\circ}$, sendo que a primeira saída lateral para a coleta do líquido situou-se após a décima calha, e assim sucessivamente.

O conjunto utilizado no ensaio foi um trator da marca VALTRA, modelo BM-120 4x2 TDA, juntamente com um turboatomizador da marca Jacto, modelo ARBUS 400 GOLDEN, composto por: tanque de $400 \mathrm{~L}$ em polietileno de alta densidade; visor de nível com escala graduada; agitador de calda por retorno hidráulico; pressão variável até $2.758 \mathrm{kPa}$; bomba JP-75, com capacidade de recalque de $75 \mathrm{~L} \mathrm{~min}^{-1}$ a $540 \mathrm{rpm}$, acionada pela tomada de potência do trator; ramal de bicos em aço inox, com 12 bicos de cada lado, com angulação individual regulável e registro individual; ventilador com pás de náilon e fibra de vidro de $0,85 \mathrm{~m}$ de diâmetro, gerando velocidade média do ar de $35 \mathrm{~m} \mathrm{~s}^{-1}$. No momento do ensaio, o turboatomizador foi equipado com pontas J5-2 e manômetro regulado na pressão de $1.378 \mathrm{kPa}$, com velocidade do ventilador de $35 \mathrm{~m} \mathrm{~s}^{1}$.

Os quatro tratamentos avaliados foram constituídos por três repetições aplicadas utilizando: com fluxo de ar e ramais com 12 pontas (CF12P); sem fluxo de ar e ramais com 12 pontas (SF12P); com fluxo de ar e ramais com seis pontas (CF6P), e sem fluxo de ar e ramais com seis pontas 
(SF6P). Para isso, o sistema de pulverização foi posicionado a uma distância de $1,20 \mathrm{~m}$ da mesa vertical e a $0,75 \mathrm{~m}$ em relação ao solo. Os funis de captura do líquido pulverizado foram dispostos a 0,$50 ; 0,75 ; 1,00 ; 1,20 ; 1,40 ; 1,65 ; 1,85 ; 2,10 ; 2,30$ e $2,55 \mathrm{~m}$ de altura em relação ao solo. O tempo de coleta do líquido pulverizado foi de 60 segundos para ambos os lados, sendo avaliados os lados esquerdo (LE) e direito (LD) do turboatomizador, utilizando como referência o deslocamento do trator. As médias dos volumes coletados nas provetas foram plotadas em gráficos no software Excel $2003^{\circledR}$, e os resultados obtidos, discutidos apenas de forma descritiva, conforme os resultados obtidos em cada altura. Para o monitoramento das condições climáticas, utilizou-se um termohigroanemômetro luxímetro digital portátil, modelo (Thal-300 ${ }^{\circledR}$ ).

\section{RESULTADOS E DISCUSSÃO}

No perfil de distribuição vertical na mesa para o lado esquerdo do turboatomizador (Figura 2), verifica-se que curvas com seis pontas, com e sem fluxo, promoveram menor volume de deposição quando comparadas às curvas com 12 pontas. Neste caso, o perfil obtido foi mais afetado pelo volume do que pelo fluxo de ar, concordando com GIL \& BADIOLA (2007) quando estudaram diferentes quantidades de bicos nos ramais, concluindo que o maior volume resultou nas maiores variações no perfil de distribuição de um turboatomizador.

Comparando as curvas do perfil de distribuição com e sem fluxo de ar e o funcionamento de todas as 12 pontas do ramal de bicos, pode-se observar que a velocidade do ar influenciou no volume de deposição, sendo que, na parte superior, houve menor volume devido a distância das pontas superiores em relação à mesa vertical. Este comportamento foi observado por LANDERS (2008), quando avaliou a variação da velocidade de ar de um turboatomizador, constatando que as gotas acompanham o fluxo de ar devido a sua velocidade, o que caracteriza que essa velocidade deveria ser variável ao longo do desenvolvimento da cultura. Em outro trabalho, LANDERS (2010) verificou que as áreas de deposição de gotas em folhas da cultura da uva eram menores no início do desenvolvimento, quando comparado próximo da colheita. Nesse sentido, concluiu que as indústrias deveriam oferecer aos agricultores a possibilidade de variar a velocidade, o fluxo e o direcionamento do ar para o dossel da cultura.

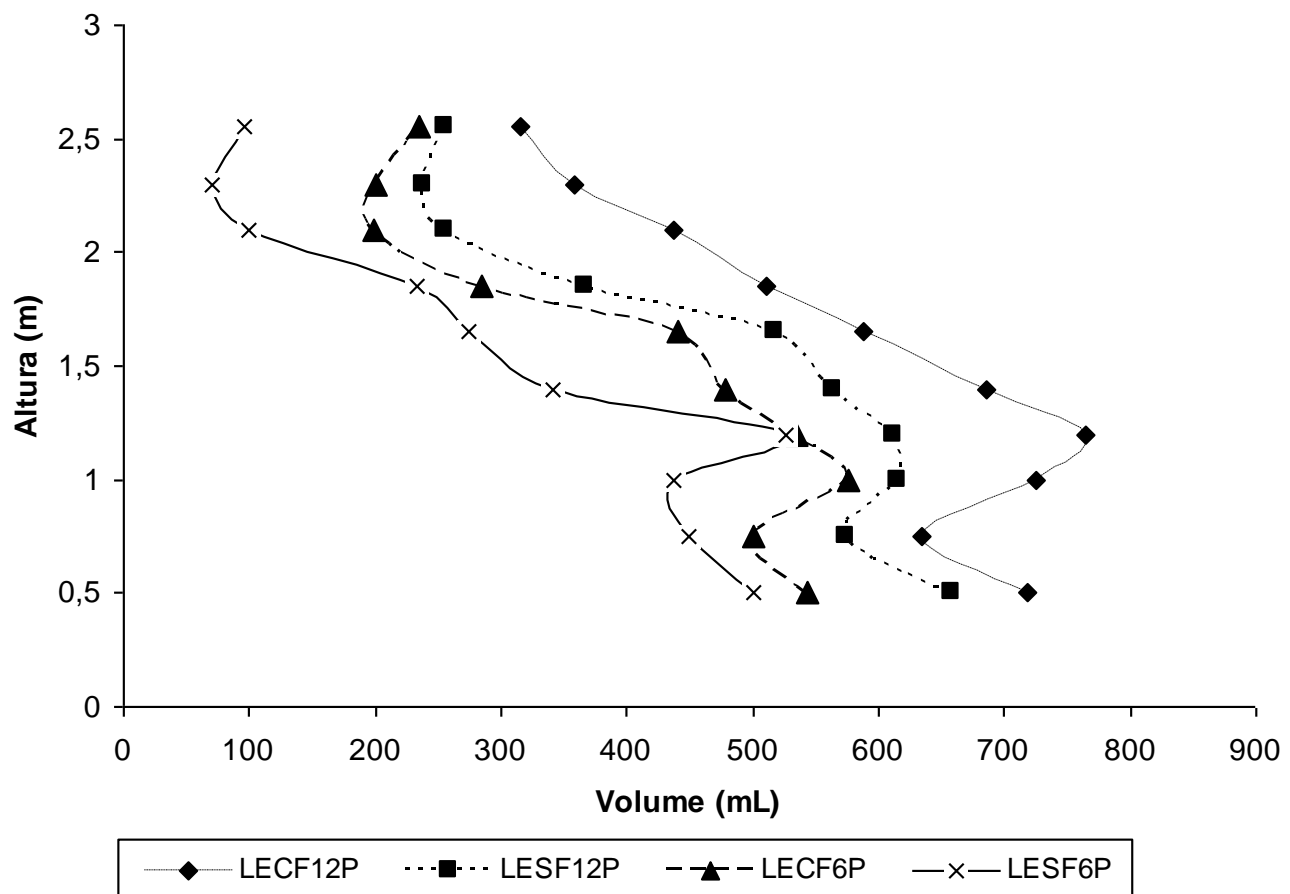

FIGURA 2. Perfil de distribuição vertical de um turboatomizador para o lado esquerdo em relação ao deslocamento do trator. Vertical distribution profile of an air assisted sprayer to the left side in relation to the tractor displacement. 
As curvas do perfil de distribuição vertical do lado direito do turboatomizador (Figura 3) também apresentaram variabilidade no padrão de distribuição, sem simetria. A variabilidade no perfil de distribuição volumétrica também foi comprovada por DANTAS (2010) quando verificou assimetria das curvas nas diferentes alturas para a cultura do mamão, com maior concentração na parte inferior da mesa. Resultados semelhantes de curvas assimétricas foram encontradas por SOMBRA et al. (2010), avaliando diferentes pressões e pontas de jato cone vazio e GIL \& BADIOLA (2007), estudando diferentes volumes de aplicações e quantidades de bicos em um turboatomizador.

Observa-se que, com fluxo de ar, quando se trabalha com seis e 12 pontas, obteve-se comportamento semelhante, apesar de a taxa de aplicação com o uso de 12 pontas ser maior. As curvas sem o fluxo de ar tiveram a mesma tendência quando se compararam seis e 12 pontas. Outra observação também constatada foi na parte superior da mesa, onde é possível observar que, nas Figuras 2 e 3, o maior volume ocorreu na parte inferior da mesa quando comparado à parte superior. Uma causa provável é a distância entre os ramais de bicos e a mesa, uma vez que, na parte inferior, encontrava-se a 1,2 m, e devido os ramais terem formato de arco, a parte superior encontrava-se a 1,75 m distante da mesa. Este comportamento demonstra que o ideal seria uma aplicação em que a distribuição não tivesse uma variabilidade ao longo do perfil de distribuição, conforme observações relatadas por LANDERS (2008), quando alterou o ângulo dos bicos, direcionando-os para a copa da cultura da uva. O mesmo autor concluiu que o direcionamento reduziu a variabilidade e melhorou a simetria da curva de distribuição na mesa vertical.

O perfil de distribuição das curvas com fluxo de ar com seis e 12 pontas obteve-se o mesmo comportamento, alterando apenas o volume coletado devido ao número de bicos utilizados. Observa-se que, na parte inferior da mesa, obteve-se maior volume quando comparado com a parte superior do lado esquerdo da mesa, sendo que, para o lado direito, a curva teve o mesmo comportamento. Pode-se observar para todos os tratamentos do lado direito, quando comparados, comportamentos semelhantes tanto com seis pontas quanto com 12 pontas, demonstrando que, neste caso, podem-se fechar as outras seis pontas que o perfil de distribuição vertical terá o mesmo comportamento, levando-se em consideração que, neste caso, o volume será alterado.

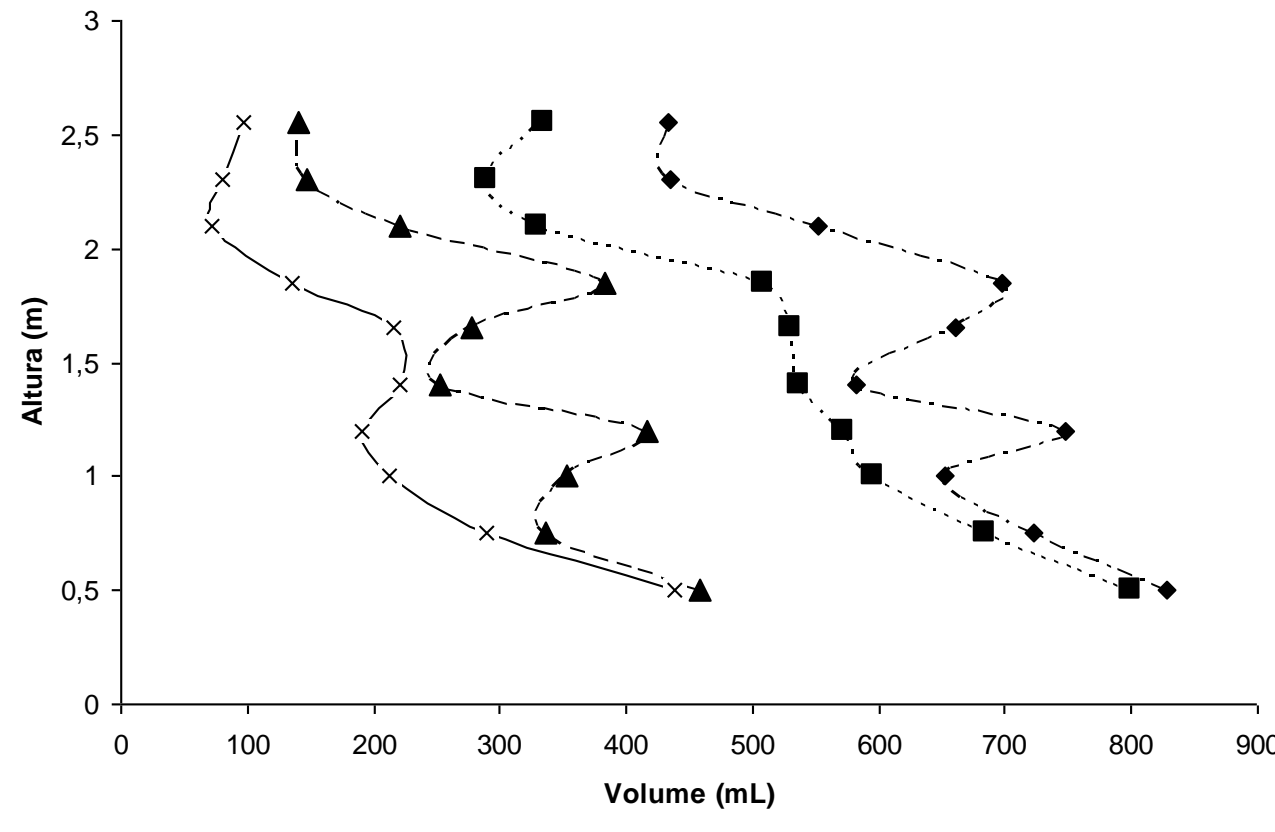

$$
\text { - - - - LDCF12P - - - - LDSF12P - - - - LDCF6P -メーLDSF6P }
$$

FIGURA 3. Perfil de distribuição vertical de um turboatomizador para o lado direito em relação ao deslocamento do trator. Vertical distribution profile of an air assisted sprayer to the right side in relation to the tractor displacement. 


\section{CONCLUSÕES}

O número de bicos não influenciou no perfil de distribuição volumétrica.

O fluxo de ar influenciou no perfil de distribuição volumétrico tanto para o lado direito quanto para o lado esquerdo da aplicação.

O turboatomizador, utilizando ou não o fluxo de ar com as 12 pontas ou as seis pontas, os maiores volumes ocorreram abaixo de $1,16 \mathrm{~m}$.

\section{REFERÊNCIAS}

ALVARENGA, C.B. de CUNHA, J.P.A.R. da. Aspectos qualitativos da avaliação de pulverizadores hidráulicos de barra na região de Uberlândia, Minas Gerais. Engenharia Agrícola, Jaboticabal, v.30, n.3, p.555-562, 2010.

BALAN, M.G.; ABI SAAB, O.J.G.; SASAKI, E.H. Distribuição da calda na cultura da Videira por turboatomizador com diferentes configurações de pontas. Ciência Rural, Santa Maria, v.36, n.3, p.731-38, 2006.

BALAN, M.G.; ABI SAAB, O.J.G.; SILVA, C.G. Depósito e perdas de calda em sistema de pulverização com turboatomizador em videira. Engenharia Agrícola, Jaboticabal, v.26. n.2, p.470477, 2006.

CUNNINGHAM, G.P.; HARDEN, J. Reducing spray volumes applied to mature citrus trees. Crop Protection, Guildford, v.17, n.4, p.289-292, 1998.

DANTAS, M.J.F. Avaliação de um sistema de aplicação de agrotóxicos na cultura do mamão: redução do risco de contaminação ambiental. 2010. 98 f. Dissertação (Mestrado em Manejo de Bacias Hidrográficas no Semiárido) - Departamento de Engenharia Agrícola, Universidade Federal do Ceará, Fortaleza, 2010.

FAROOQ, M.; LANDERS, A.J. Interactive Effects of Air, Liquid and canopies on Spray Patterns of Axial-flow Sprayers. In: 2004 ASAE/CSAE ANNUAL INTERNATIONAL MEETING, Ottawa, Ontario, Canada. St. Joseph: ASAE, 2004. (Paper 041001)

GIL, E.; BADIOLA, J. Design and verification of a portable vertical patternator for vineyard sprayer calibration. Applied Engineering in Agriculture, St. Joseph, v.23, n.1, p.35-42, 2007.

HOLOWNICKI, R.; DORUCHOWSKI, G.; SWIECHOWSKI, W.; GODYN, A. Automatic self adjusting air-jet sprayer concept for fruit trees. In: INTERNATIONAL CONFERENCE ON AGRICULTURAL ENGINEERING, 2000, Warwick. Paper... 10 p. (Paper 00053).

LANDERS, A.J. Developments towards an automatic precision sprayer for fruit crop canopies. St. Joseph: ASABE, 2010. (Paper, 1008973).

LANDERS, A.J.; FAROOQ, M. Factors influencing air and pesticide penetration into grape vine canopies. Aspects of Applied Biology, v.71, p.343-348, 2004.

LANDERS A.J. Airblast sprayers. In: PIMENTEL, D. (Ed.). Encyclopedia of pest management. New York: Marcel Dekker, 2002a. p.11-13.

LANDERS, A.J. Improving spraying efficiency. Disponível em: <www.nysaes.cornell.edu/ent/faculty/landers/pestapp.2008>. Acesso em: 23 jun. 2010.

LANDERS A.J. The answer is blowing in the wind. Aspects of Applied Biology. International Advances in Pesticide Application, v.66, p.177-184, 2002b.

LANDERS, A.J.; GIL, E. Development and validation of a new deflector system to improve pesticide application in New York and Pennsylvania grape production areas. In:

INTERNATIONAL CONFERENCE ON AGRICULTURAL ENGINEERING, 2006, Warwick. Paper... 10 p. (Paper 061001). 
PAI, N.; SALYANI, M.; SWEEB, R.D. Regulating airflow of orchard airblast sprayer based on tree foliage density. Transactions of the ASABE, St. Joseph, v.52, n.5, p.1.423-1.428, 2009.

PRAT, M.I.H.; TEIXEIRA, M.M.; RODRIGUES, G.J.; MILÁN, H.; LAS, C. Modelación mediante la dinámica de flujo computacional de la corriente de aire de un pulverizador. Revista Ciencias Técnicas Agropecuarias, La Habana, v.15, n.1, p.12-18, 2006.

SINDAG 2009. SINDICATO NACIONAL DA INDÚSTRIA DE PRODUTOS PARA DEFESA AGRÍCOLA. Disponível em: http://www.sindag.com.br>. Acesso em: 20 nov. 2009.

SOMBRA, W.A.; MION, R.L.; DANTAS, M.J.F.; VILLIOTI, C.A.; NASCIMENTO, E.M.S. Avaliação do perfil vertical de distribuição volumétrica de um turboatomizador. In: FURLANI, C.E.A.; SILVA, R.P. da; ROSALEN, D.L.; SILVA, J.G.F. da; REIS, E.F. dos; VENTURIN, J.B.A. Engenharia Agrícola e o desenvolvimento das propriedades familiares. Jaboticabal: Associação Brasileira de Engenharia Agrícola, 2010. 1 CD-ROM. 